\title{
Intravoxel incoherent motion (IVIM) in diffusion-weighted imaging (DWI) for Hepatocellular carcinoma: correlation with histologic grade
}

\author{
Vincenza Granata ${ }^{1}$, Roberta Fusco $^{1}$, Orlando Catalano ${ }^{1}$, Benedetta Guarino ${ }^{1}$, \\ Francesco Granata2, Fabiana Tatangelo ${ }^{3}$, Antonio Avallone ${ }^{4}$, Mauro Piccirillo ${ }^{5}$, \\ Raffaele Palaia ${ }^{5}$, Francesco Izzo ${ }^{5}$, Antonella Petrillo ${ }^{1}$ \\ ${ }^{1}$ Department of Radiology, "Istituto Nazionale Tumori IRCCS Fondazione Pascale - IRCCS di Napoli", Naples, I-80131, Italy \\ ${ }^{2}$ Department of Civil and Mechanical Engineering, "Università degli Studi di Cassino e del Lazio Meridionale", Cassino \\ 03043, Italy \\ ${ }^{3}$ Departement of Pathology, "Istituto Nazionale Tumori IRCCS Fondazione Pascale - IRCCS di Napoli", Naples, I-80131, Italy \\ ${ }^{4}$ Department of Gastrointestinal Oncology, "Istituto Nazionale Tumori IRCCS Fondazione Pascale - IRCCS di Napoli", Naples, \\ I-80131, Italy \\ ${ }^{5}$ Department of Hepatobiliary Surgical Oncology, "Istituto Nazionale Tumori IRCCS Fondazione Pascale - IRCCS di Napoli", \\ Naples, I-80131, Italy
}

Correspondence to: Roberta Fusco, email: r.fusco@istitutotumori.na.it

Keywords: HCC, magnetic resonance imaging, diffusion weighted imaging, histologic grade

Received: July 06, $2016 \quad$ Accepted: September 29, $2016 \quad$ Published: October 15, 2016

\section{ABSTRACT}

Purpose: To assess the correlation between DWI diffusion parameters obtained using Intravoxel Incoherent Motion Method (IVIM) and histological grade of Hepatocellular carcinoma (HCC).

Results: According to Edmondson-Steiner grade lesions were classified with grade 1 (14), grade 2 (30), grade 3 (18), and grade 4 (0). Apparent Diffusion Coefficient (ADC), perfusion fraction ( $f p)$, tissue diffusion coefficient (Dt) median values were statistically different in HCC groups with 1, 2, 3 histological grade $(\mathrm{p}<0.001)$. A significant correlation was reported between ADC, $\mathrm{fp}$, Dt and histologic grade respectively of $0.687,0.737$ and 0.674 . Receiver operating characteristic (ROC) analysis demonstrated that an ADC of $2.11 \times 10-3 \mathrm{~mm}^{2} / \mathrm{sec}_{\text {, }}$ an $\mathrm{fp}$ of $47.33 \%$ and an Dt of $0.94 \times 10-3 \mathrm{~mm}^{2} / \mathrm{sec}$ were the optimal cutoff values to differentiate high histological grade (3) versus low histological grade (1-2), with a sensitivity and specificity for ADC of $100 \%$ and $100 \%$, for $\mathrm{fp}$ of $100 \%$ and $89 \%$, for Dt of $100 \%$ and $74 \%$, respectively.

Material and Methods: A retrospective approved study was performed including 34 patients with 62 HCCs. IVIM was performed to obtain ADC, fp, pseudo-diffusion coefficient (Dp), Dt coefficients. Kruskal Wallis, Spearman Correlation Coefficient, ROC analysis were performed.

Conclusions: ADC and IVIM-derived fp showed significantly better diagnostic performance in differentiating high-grade from low-grade $\mathrm{HCC}$, and significant correlation was observed between ADC, fp, Dt and histological grade.

\section{INTRODUCTION}

Hepatocellular carcinoma (HCC) is one of the most common human solid tumors worldwide $[1,2]$. European Association for the Study of the Liver (EASL), in accordance with the guidelines of the American Association for the Study Liver Diseases (AASLD), recommended that in order to characterize $\mathrm{HCC}$, invasive criteria by imaging techniques such as multidetector Computed Tomography
(CT) and Dynamic Contrast enhanced Magnetic Resonance Imaging (DCE-MRI) can be utilized only in cirrhotic patients [3]. Diagnosis should be based on the identification of the typical hallmark of HCC (hypervascular in the arterial phase with washout in the portal venous or delayed phases) [3]. Although the MRI is the technique which best suits to characterize and to follow HCC patients $[4,5]$, no agreement regarding the study protocol and the impact of Diffusion-weighted imaging (DWI) has been found. DWI 
is capable of evaluating the mobility of water proton and can provide information on microstructural organization of a tissue, the cell density, the integrity of cell membrane and its vitality $[6,7]$. During 1986, Le Bihan, was the first, to evaluate intravoxel incoherent motion (IVIM) as an analytical approach to characterize the link between the tissues signal attenuation and the increase in the $b$ value, and thanks to biexponential model, it was possible to separate the diffusion of water molecules to microcapillary perfusion of tissues [8]. IVIM data can be evaluated either quantitatively or qualitatively; quantitative data may be useful for tissue characterization and functional assessment, while qualitative evaluation may be useful to identify disease [9]. In clinical practice, DWI is used to assess treatment response of liver lesions after neoadjuvant therapy [10] or ablative techniques [11]. Another interesting field is the correlation between the quantitative and semi-quantitative values of DWI with histological grade of the tumor, as was seen in bladder [12], endometrial [13], breast [14], and brain cancers [15]. Recent studies, also in HCC, had evaluated the correlation between DWI and histological grade [16-19] considering that the $\mathrm{HCC}$ histologic grade is an important recurrence and survival predictive factor after hepatic resection and transplantation: poorly differentiated HCC are associated to worse survival in comparison of well and moderately differentiated HCC
[20]. The possibility that imaging study can correlate to histologic grade to select the therapeutic strategy would be of great value in helping to direct the proper management of HCC. Therefore, our purpose was to test if DWI and IVIM parameters could correlate with histological grade of HCC and became a prognostic imaging factors.

\section{RESULTS}

A total of $62 \mathrm{HCCs}$ were evaluated (median 1.82 lesions; diameter between 12 and $20 \mathrm{~mm}$ ). All tumors were histologically classified according to the major Edmondson-Steiner grade on final pathologic reports as follows: 14 with grade 1, 30 with grade 2, 18 with grade 3 , and no one with grade 4 . Figure 1, 2 and 3 report example of HCC of grade 1, 2 and 3 respectively.

Table 1 reports mean value \pm standard deviation for ADC ad IVIM parameters for different histological grade. Mean values of ADC, fp, Dt were statistically different in $\mathrm{HCC}$ groups with 1, 2, 3 histological grade ( $\mathrm{p}$ value $<0.001$ at test of Kruskal Wallis).

Correlation coefficient obtained according to Spearman's rank was performed for each couple of parameters and was reported in Table 2. A significant correlation was reported between $\mathrm{ADC}$, fp, Dt and histologic

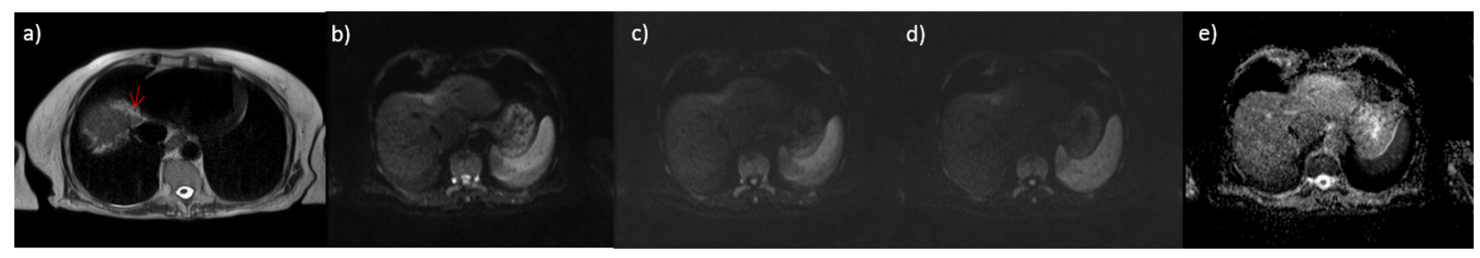

Figure 1: small HCC on IV hepatic segment G1; in a. HASTE T2-wejgthed image, in b. DWI image at b value $50 \mathrm{~s} / \mathrm{mm}^{2}$, in c. DWI image at b value $400 \mathrm{~s} / \mathrm{mm}^{2}$, in d. DWI image at b value $800 \mathrm{~s} / \mathrm{mm}^{2}$ and in e. ADC map.

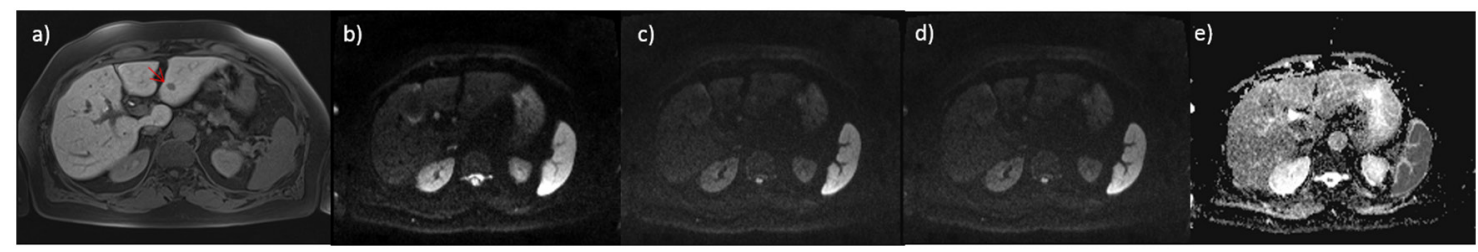

Figure 2: small HCC on III hepatic segment G2; in a. VIBE T1 weighted image, in b. DWI image at b value $50 \mathrm{~s} / \mathrm{mm}^{2}$, in c. DWI image at b value $400 \mathrm{~s} / \mathrm{mm}^{2}$, in d. DWI image at b value $800 \mathrm{~s} / \mathrm{mm}^{2}$ and in e. ADC map.

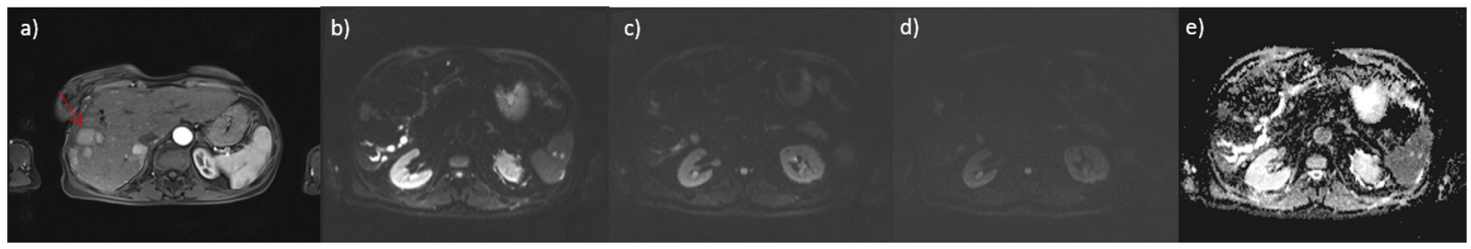

Figure 3: HCC on V hepatic segment G3; in a. VIBE T1 weighted image, in b. DWI image at b value $50 \mathrm{~s} / \mathrm{mm}^{2}$, in c. DWI image at b value $400 \mathrm{~s} / \mathrm{mm}^{2}$, in d. DWI image at b value $800 \mathrm{~s} / \mathrm{mm}^{2}$ and in e. ADC map. 
Table 1: ADC and IVIM derived diffusion and perfusion parameters: mean and standard deviation values according to different histological grade of HCC

\begin{tabular}{lccccc}
\hline Grading & & $\begin{array}{c}\text { ADC } \\
{\left[\mathbf{m m}^{2} / \mathbf{s ~ 1 0} \mathbf{~}^{-3}\right]}\end{array}$ & $\begin{array}{c}\mathbf{f p} \\
{[\mathbf{\%}]}\end{array}$ & $\begin{array}{c}\mathbf{D p} \\
{\left[\mathbf{m m}^{2} / \mathbf{s ~ 1 0} \mathbf{1 0}^{-3}\right]}\end{array}$ & $\begin{array}{c}\mathbf{D t} \\
{\left[\mathbf{m m}^{2} / \mathbf{s} \mathbf{1 0}^{-3}\right]}\end{array}$ \\
\hline $\mathbf{1}$ & Mean & $\mathbf{0 , 9 4}$ & $\mathbf{1 4 , 4 8}$ & $\mathbf{5 4 , 6 9}$ & $\mathbf{0 , 8 6}$ \\
$\mathbf{2}$ & Standard deviation & 0,12 & 7,38 & 83,62 & 0,09 \\
& Mean & $\mathbf{1 , 0 3}$ & $\mathbf{3 1 , 4 9}$ & $\mathbf{4 4 , 4 9}$ & $\mathbf{0 , 8 1}$ \\
$\mathbf{3}$ & Standard deviation & 0,31 & 17,60 & 70,15 & 0,49 \\
& Mean & $\mathbf{2 , 4 4}$ & $\mathbf{5 4 , 0 8}$ & $\mathbf{1 3 , 7 0}$ & $\mathbf{1 , 5 1}$ \\
Total & Standard deviation & 0,04 & 2,96 & 7,39 & 0,11 \\
& Mean & 1,42 & 34,21 & 36,78 & 1,03 \\
P value* & Standard deviation & 0,70 & 19,25 & 61,45 & 0,46 \\
\hline
\end{tabular}

*Kruskal-Wallis Test

Table 2: ADC and IVIM derived diffusion and perfusion parameters: Spearman Correlation Coefficient for each couple of parameters

\begin{tabular}{|c|c|c|c|c|c|c|c|}
\hline & & & GRADING & ADC & fp & Dp & Dt \\
\hline \multirow[t]{10}{*}{$\begin{array}{l}\text { Spearman } \\
\text { Correlation } \\
\text { Coefficient }\end{array}$} & GRADING & $\begin{array}{l}\text { Correlation } \\
\text { Coefficient }\end{array}$ & 1,000 &, $687^{*}$ &, $737^{*}$ &,- 326 &, $674^{*}$ \\
\hline & & P value & &, 000 & ,000 & ,090 &, 000 \\
\hline & ADC & $\begin{array}{l}\text { Correlation } \\
\text { Coefficient }\end{array}$ &, $687^{*}$ & 1,000 &, $483^{*}$ &,- 128 &, $835^{*}$ \\
\hline & & $P$ value &, 000 & &, 006 &, 518 &, 000 \\
\hline & fp & $\begin{array}{l}\text { Correlation } \\
\text { Coefficient }\end{array}$ &, $737^{*}$ &, $483^{*}$ & 1,000 &,$- 615^{*}$ & ,245 \\
\hline & & $P$ value &, 000 & ,006 & & ,001 & ,183 \\
\hline & Dp & $\begin{array}{l}\text { Correlation } \\
\text { Coefficient }\end{array}$ &,- 326 &,- 128 &,$- 615^{*}$ & 1,000 & ,290 \\
\hline & & $P$ value & ,090 & ,518 & ,001 & & ,135 \\
\hline & Dt & $\begin{array}{l}\text { Correlation } \\
\text { Coefficient }\end{array}$ & ,674* &, $835^{*}$ & 245 & ,290 & 1,000 \\
\hline & & $P$ value & ,000 &, 000 & ,183 & ,135 & \\
\hline
\end{tabular}

*A p value $<0.05$ was considered statistically significant.

grade of HCC respectively of $0.687,0.737$ and 0.674 with a significant statistically difference ( $\mathrm{p}$ value $<0.05$ ).

ROC analyses demonstrated that an ADC of $2.11 \times 10^{-3} \mathrm{~mm}^{2} / \mathrm{sec}$, an fp of $47.33 \%$ and an Dt of $0.94 \times 10^{-3}$ $\mathrm{mm}^{2} / \mathrm{sec}$ were the most accurate cutoff levels (calculated with Youden index) to differentiate high histological grade (3) versus low histological grade (1 and 2), with a sensitivity and specificity for ADC of $100 \%$ and $100 \%$, for fp of $100 \%$ and $89 \%$, for Dt of $100 \%$ and $74 \%$, respectively.

In addition, ADC was shown to have the best diagnostic performance in comparison of $\mathrm{fp}$ and $\mathrm{Dt}$ for differentiating high from low grade of HCC (grade 3 versus and 2), with a corresponding area under the ROC curve of 1.0 ( $\mathrm{p}$ value $<0.001), 0.90(\mathrm{p}$ value $=0.05)$ and $0.95(\mathrm{p}$ value $=0.036)($ Table 3$)$. 
Table 3: ADC and IVIM derived diffusion and perfusion parameters: Area under ROC curve with optimal cutoff values, sensitivity and specificity

\begin{tabular}{lccccc}
\hline & Area & Cutoff value & pvalue & $\begin{array}{c}\text { Sensitivity } \\
{[\%]}\end{array}$ & $\begin{array}{c}\text { Specificity } \\
{[\%]}\end{array}$ \\
\hline ADC $\left[\mathbf{m m}^{2} / \mathbf{s e c}\right]$ & 1,000 & $2.11 \times 10^{-3}$ &, 000 & 100 & 100 \\
$\mathbf{f p}[\%]$ &, 895 & 47,33 &, 000 & 100 & 89 \\
Dp $\left[\mathbf{m m}^{2} / \mathbf{s e c}\right]$ &, 327 & $18.3 \times 10^{-3}$ &, 038 & 56 & 43 \\
$\mathbf{D t}\left[\mathbf{m m}^{2} / \mathbf{s e c}\right]$ &, 947 & $0.94 \times 10^{-3}$ &, 000 & 100 & 74 \\
\hline
\end{tabular}

\section{DISCUSSION}

The accurate distinction of well-differentiated HCCs from less well-differentiated HCCs is considered an important issue in planning the therapeutic strategy [23]. Considering that histological confirmation of small suspicious hepatic nodules before treatment is often not possible owing to their location in the liver, the role of pre-operative imaging technique for the discrimination of well, moderate and poorly differentiated HCCs is important [24]. Our results suggested that DWI could be used to predict the histological grade of $\mathrm{HCC}$, in fact there was a good correlation between ADC and grading, between fp and grading, and between Dt and grading. Nakanishi et al, in previous study, demonstrated not only the usefulness of DWI for histological tumor grading, but also the possibility to use ADC as an early predictive factor in pre-surgical phase of HCC recurrence in the six months following surgery [25]. DWI is a valuable diagnostic tool, that using water mobility assessment reflects indirectly tissue biological characteristics [12-19, 25]. Water mobility is restricted in malignant tissue due to increase of cellular density and decrease of interstitial space; this translates to signal hyperintensity in diffusion weighted images and signal hypointensity in an ADC map [7]. In a recent meta-analysis was reported the DWI performance in the prediction of HCC histological grade: Chen et al showed that in the discrimination between well differentiated HCC by higher grades, DWI had a sensitivity of $54 \%$, a specificity of $90 \%$, and an area under ROC curve 0.9311 . While, for differentiating poorly differentiated HCC from lower grades, the sensitivity was $84 \%$, specificity was $48 \%$, and a moderately high diagnostic performance $(\mathrm{AUC}=0.8513)[18]$. According to Chen, in our study, ROC analyses demonstrated that an ADC of $2.11 \times 10^{-3} \mathrm{~mm}^{2} / \mathrm{sec}$, an $\mathrm{fp}$ of $47.33 \%$ and an Dt of $0.94 \times 10^{-3} \mathrm{~mm}^{2} / \mathrm{sec}$ were the most accurate cutoff levels to differentiate high histological grade (3) versus low histological grade (1 and 2), with a sensitivity and specificity for ADC of $100 \%$ and $100 \%$, for fp of $100 \%$ and $89 \%$, for Dt of $100 \%$ and $74 \%$, respectively. Nasu et al, in a series of 125 resected HCCs (sizes range: $0.8-15$ $\mathrm{cm}$ ), found no correlation between histological grade and $\mathrm{ADC}$ (using $\mathrm{b}$ factors of 0 and $500 \mathrm{smm}^{2}$ ), although the
DWI and Signal Intensity (SI) of the HCCs increased in higher grade [26]. Instead, Muhi et al showed significant differences both in SI and ADC values (using $b$ factors of 500 and $1000 \mathrm{smm}^{2}$ ) between various grades of 98 HCC (size range, 0.8-5.3 cm), although there was still considerable overlapping [27]. Nishie et al demonstrated a correlation of ADC value with the HCCs histological grade, but the difference was significant only between the extreme groups (between well-differentiated HCCs and poorly differentiated HCCs) [28]. Conversely, to Nishie, in our series, there was a linear proportional tendency for ADC, fp and Dt values according to the transition from well-differentiated to poorly differentiated. Current technique improvement has supported the IVIM usein the prediction of the HCC histological grade. IVIM approach provides both estimate of water pure mobility and a measure of microscopic blood diffusion in capillaries network. [8-9]. Pure diffusion coefficient (Dt) derived by IVIM model according to Woo et al had significantly better diagnostic accuracy than apparent diffusion coefficient to differentiate low and high HCC histological grade [17]. Conversely in our study, the best diagnostic performance was obtained by ADC in comparison of $\mathrm{fp}$ and $\mathrm{Dt}$ for differentiating high from low grade HCC (grade 3 versus 1 and 2), with a corresponding area under the ROC curve of 1.0 ( $\mathrm{p}$ value $<0.001), 0.90(\mathrm{p}$ value $=0.05)$ and $0.95(\mathrm{p}$ value $=0.036$ )

This study has a limitation. To obtain accurate informations about tissue perfusion proprieties by IVIM model applied on DWI data in need to acquire imaging using lower $b$ values $\left(<100-200 \mathrm{~s} / \mathrm{mm}^{2}\right)$ and an adequate total number of $b$ values. Conversely, there is no consensus on number and amplitude of the $b$ values that should be used in clinical setting. The magnitude of $b$ values differs between literature studies and the number ranges from 4 to more than 10 . On the other hand, different b-values groupings may cause bias and variability estimation of IVIM parameters, especially in IVIM-derived fp [29]. Koh et al [30] suggested six to eight b values in total, with four or more within the perfusion-sensitive range $(b$ $<100 \mathrm{~s} / \mathrm{mm}^{2}$ ) to better quantify pseudo-diffusion motion. Due to the restriction of the used MR equipment which does not allow to use $b$ values with an interval of less than $50 \mathrm{~ms}$, we have utilized only $3 \mathrm{~b}$ values between 0 and 
$100\left(0,50\right.$ and $\left.100 \mathrm{~s} / \mathrm{mm}^{2}\right)$. To obviate this constraint we used a special algorithm to calculate IVIM parameters that Fusco et al [22] demonstrated to perform better than conventional Levenberg Marquardt (LM) algorithm for IVIM parameters estimation. Fusco et al in [22] examined the performance of LM and VARPRO for IVIM estimation on a simulation basis and concluded that VARPRO technique had smaller bias and better fitting with respect to LM; this latter was demonstrated on simulation data in term of residual sum of square (RSS) both for single search start point and for multiple search start point approach.

HCC histologic grade is a important factor to predict recurrence and survival after hepatic resection and transplantation. The possibility that imaging study can correlate to histologic grade to selecting the therapeutic strategy would be of value in helping to direct the proper management in patients with HCC. In this context, MR study with the DWI sequences is the method of choice because the ADC and IVIM-derived fp values is related to the histological grade of $\mathrm{HCC}$, presented a better diagnostic performance to differentiate low and high HCC histological grade.

\section{MATERIALS AND METHODS}

\section{Patient population}

A retrospective study, approved by National Cancer Institute Pascale Foundation of Naples, was performed through a computerized search of medical records on 74 patients underwent liver MR imaging and followed by biopsy for HCC from August 2014 to February 2016. After reviewing the medical records, 18 patients were excluded because the final pathology report was confirmed not to be HCC; 14 patients because the tumors were smaller than $1 \mathrm{~cm}$ and 8 were excluded due to patient movement or image noise.

The final study population included 34 patients (8 women and 26 men; mean age 72 years; range: 5683 years) with 62 HCCs. The mean interval standard deviation between pathologic examination and MR imaging was 15 days (range 4-28 days). All patients had chronic liver disease which was related to hepatitis C virus infection in 14 patients, hepatitis B virus in 18 cases, and alcohol abuse in 2; all were stage A according to the Barcelona-Clinic Liver Cancer (BCLC) classification; alpha-fetoprotein levels were $>4 \mathrm{ng} / \mathrm{ml}$ (12-320 ng/ml, mean $80 \mathrm{ng} / \mathrm{ml}$ ) in all patients.

\section{MR imaging protocol}

MR imaging was performed by using a $1.5 \mathrm{~T}$ MR (Magnetom Symphony, with Total Imaging Matrix Package, Siemens, Erlangen, Germany) with 8-element body and phased array coils. The MRI examination consisted of basal images taken before IV administration of contrast medium and then functional dynamic sequences obtained after IV injection of liver-specific contrast medium, acquiring the last series of images with a delay of 20 minutes during the hepatobiliary excretion of the contrast medium. The baseline sequences obtained before IV contrast medium were coronal Trufisp T2weighted free breathing; axial Half-Fourier Acquisition Single-Shot Turbo Spin-Echo (HASTE) T2-weighted, with controlled respiration, without and with fatsuppressed (FS) gradient-echo pulse; coronal HASTE T2-weighted, without FS; axial flash in-out phase T1-weighted, with controlled respiration; Volumetric Interpolated Breath-hold Examination (VIBE) T1weighted SPAIR with controlled respiration; diffusion weighted imaging (DWI) with planar echo-pulse sequence (EPI) at several $\mathrm{b}$ value $b$ value $0,50,100$, $200,400,600$, and $800 \mathrm{~s} / \mathrm{mm}^{2}$. As contrast medium the liver-specific gadolinium ethoxybenzyl dimeglumine (Primovist, Bayer Schering Pharma, Germany) was employed All patients received $0.1 \mathrm{ml} / \mathrm{kg}$ of Primovist by means of a power injector (Spectris Solaris ${ }^{\circledR}$ EP MR, MEDRAD Inc., Indianola, IA, USA), at an infusion rate of $1 \mathrm{ml} / \mathrm{s}$. After completion of IV contrast medium administration, VIBE T1-weighted FS (SPAIR) sequences were acquired in five different phases: hepatic arterial (35 s delay), portal venous (70 s), equilibrium (90 s), delayed (120 s), hepatobiliary excretion (20 minutes). Details of sequence parameters were reported in Table $4[10,21]$.

\section{Images analysis}

In this study the diffusion parameters estimation was performed using the intravoxel incoherent motion method $[8,9]$.

Bi-exponential model to estimate the IVIM-related parameters of pseudo-diffusivity $\left(\mathrm{D}_{\mathrm{p}}\right)$, perfusion fraction $\left(f_{p}\right)$, and tissue diffusivity $\left(D_{t}\right)$ was described by the following equation

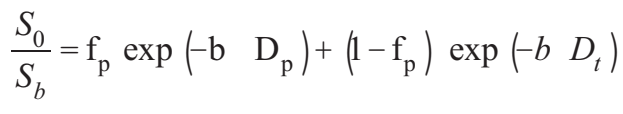

We used a VARiable PROjection approach to estimate the three parameters because the bi-exponential model may often be ill-conditioned because of a limited number of samples, small perfusion fraction and/or similar compartmental diffusivities. In a previous study we have demonstrated that the VARiable PROjection algorithm is superior to the conventional Levenberg-Marquardt algorithm for non linear curve fitting in intravoxel incoherent motion method for DW-MRI data analysis [22]. A brief explanation of VARPRO approach is described in the following. 
Table 4: Pulse Sequence Parameters on MR studies

\begin{tabular}{|c|c|c|c|c|c|c|}
\hline Sequence & Orientation & $\begin{array}{c}\text { TR/TE/FA } \\
\text { (ms/ms/deg.) }\end{array}$ & $\begin{array}{c}\text { AT } \\
\text { (min.) }\end{array}$ & $\begin{array}{c}\text { Acquisition } \\
\text { Matrix }\end{array}$ & $\begin{array}{l}\text { Slice thickness/ } \\
\text { Gap }(\mathrm{mm})\end{array}$ & Fat Suppression \\
\hline $\begin{array}{l}\text { TRUFISP } \\
\text { T2-W }\end{array}$ & Coronal & $4.30 / 2.15 / 80$ & 0.46 & $512 \times 512$ & $4 / 0$ & without \\
\hline HASTE T2-W & Axial & $1500 / 90 / 170$ & 0.36 & $320 \times 320$ & $5 / 0$ & $\begin{array}{l}\text { Without and with } \\
\text { (SPAIR) }\end{array}$ \\
\hline HASTE T2w & Coronal & $1500 / 92 / 170$ & 0.38 & $320 \times 320$ & $5 / 0$ & without \\
\hline In-Out phase $\mathrm{T} 1-\mathrm{W}$ & Axial & $160 / 2.35 / 70$ & 0.33 & $256 \times 192$ & $5 / 0$ & without \\
\hline DWI & Axial & $7500 / 91 / 90$ & 7 & $192 \times 192$ & $3 / 0$ & without \\
\hline $\begin{array}{l}\text { VIBE } \\
\text { T1-W }\end{array}$ & Axial & $4.80 / 1.76 / 12$ & 0.18 & $320 \times 260$ & $3 / 0$ & with (SPAIR) \\
\hline
\end{tabular}

Note $-\mathrm{W}=$ Weighted, $\mathrm{TR}=$ Repetition time, $\mathrm{TE}=$ Echo time, $\mathrm{FA}=$ Flip angle, $\mathrm{AT}=$ Acquisition time, $\mathrm{SPAIR}=\mathrm{Spectral}$ Adiabatic Inversion Recovery, HASTE $=$ Half-Fourier acquisition single-shot turbo spin-echo, DWI = Diffusion-weighted imaging VIBE $=$ Volumetric interpolated breath hold examination

Rearranging the equation (1) the $S(b) / S_{0}-e^{-b D_{t}}$ is the product of $f$ and a nonlinear function of $\mathrm{Dt}$ and $\mathrm{Dp}$ :

$$
\mathrm{f}\left(\mathrm{D}_{\mathrm{p}} ; \mathrm{D}_{\mathrm{t}} ; \mathrm{b}\right)=S(b) / S_{0}-e^{-b D_{t}}=f\left(e^{-b D_{p}}-e^{-b D_{t}}\right)
$$

Letting $\mathrm{f}\left(\mathrm{D}_{\mathrm{p}} ; \mathrm{D}_{\mathrm{t}} ; \mathrm{b}\right)$ the cost functional becomes:

$$
S(b) / S_{0} .-e^{-b D_{t}}=\left\|\mathrm{y}-\mathrm{f}\left(\mathrm{D}_{\mathrm{p}} ; \mathrm{D}_{\mathrm{t}} ; \mathrm{b}\right) \mathrm{f}\right\|_{2}
$$

Therefore, a separable nonlinear least square model known as VARiable PROjection (VARPRO) can be used to calculate the diffusion parameters. If we knew, the estimate of the nonlinear parameters Dp and Dt the estimate of the linear parameter f could be obtained by:

$$
f=e^{-b D_{t}}+\mathrm{y}+\mathrm{f}\left(\mathrm{D}_{\mathrm{p}} ; \mathrm{D}_{\mathrm{t}} ; \mathrm{b}\right)^{+}
$$

where $f\left(D_{p} ; D_{t} ; b\right){ }^{+}$is the Moore-Penrose generalized inverse of $\mathrm{f}\left(\mathrm{D}_{\mathrm{p}} ; \mathrm{D}_{\mathrm{t}} ; \mathrm{b}\right)$. Therefore, a new cost functional can be constructed:

$$
S(b) / S_{0} \cdot-e^{-b D_{t}}=\left\|\mathrm{y}-\mathrm{f}\left(\mathrm{D}_{\mathrm{p}} ; \mathrm{D}_{\mathrm{t}} ; \mathrm{b}\right) \mathrm{f}\left(\mathrm{D}_{\mathrm{p}} ; \mathrm{D}_{\mathrm{t}} ; \mathrm{b}\right)^{+} \mathrm{y}\right\|_{2}
$$

This analysis was ROI-based using median value of single voxel signals for each $b$ value. ROIs for the tumor were manually drawn to include such hyperintense voxels on image at b value $800 \mathrm{~s} / \mathrm{mm}^{2}$. No motion correction algorithm was used but ROIs were drawn taking care to exclude areas in which movement artifacts or blurring caused voxel misalignments.

The data analysis was performed using an in-house software written in Matlab (The MathWorks, Inc., Natick, MA, USA).

\section{Statistical analysis}

Data were expressed in terms of median value \pm standard deviation. Kruskal Wallis non-parametric test was performed to emphasize significant statistically difference between median value of ADC, Dp, fp and Dt for different grading subgroups.

The Spearman rank correlation test was used to compare the mean ADC and IVIM parameter values according to Edmondson-Steiner grade. The correlation coefficient rho (r) was obtained to compare the degree of correlation as follows: little or no relationship if $0 \leq \mathrm{r}<$ 0.25 , fair if $0.25 \leq \mathrm{r}<0.5$, moderate to good if $0.5 \leq \mathrm{r}<$ 0.75 , and very good to excellent if $0.75 \leq \mathrm{r}$.

Receiver operating characteristic (ROC) curves were used to determine whether ADC and IVIM-derived parameters could be used to discriminate between low grade (1-2) and high grade (3) HCC. Then, sensitivity and specificity for predicting high histologic grade were calculated according to the cutoff value that demonstrated the greatest Youden index on estimated curves. The area under the ROC curve was obtained to compare their diagnostic capacities.

A $p$ value $<0.05$ was considered statistically significant.

All analyses were performed using Statistics Toolbox of Matlab R2007a (The Math-Works Inc., Natick, MA).

\section{ACKNOWLEDGMENTS}

The authors are grateful to Alessandra Trocino, librarian at the National Cancer Institute of Naples, Italy. 
Moreover, for the collaboration, authors are grateful to Maria Bruno, Laura Galeani, Rita Guarino, and Assunta Zazzaro.

\section{CONFLICTS OF INTEREST}

Authors declare no conflicts of interest.

\section{REFERENCES}

1. Izzo F, Palaia R, Albino V, Amore A, di Giacomo R, Piccirillo M, Leongito M, Nasto A, Granata V, Petrillo A, Lastoria S. Hepatocellular carcinoma and liver metastases: clinical data on a new dual-lumen catheter kit for surgical sealant infusion to prevent perihepatic bleeding and dissemination of cancer cells following biopsy and locoregional treatments. Infect Agent Cancer 2015; 10: 11.

2. Piccirillo M, Granata V, Albino V, Palaia R, Setola SV, Petrillo A, Tatangelo F, Botti G, Foggia M, Izzo F. Can hepatocellular carcinoma (HCC) produce unconventional metastases? Four cases of extrahepatic HCC. Tumori 2013; 99: e19-23.

3. European Association for Study of Liver; European Organisation for Research and Treatment of Cancer. EASL-EORTC clinical practice guidelines: management of hepatocellular carcinoma. Eur J Cancer; 48: 599-641. Erratum in: Eur J Cancer; 48: 1255-6.

4. Granata V, Petrillo M, Fusco R, Setola SV, de Lutio di Castelguidone E, Catalano O, Piccirillo M, Albino V, Izzo F, Petrillo A. Surveillance of HCC Patients after Liver RFA: Role of MRI with Hepatospecific Contrast versus ThreePhase CT Scan-Experience of High Volume Oncologic Institute. Gastroenterol Res Pract 2013; 2013: 469097.

5. Di Martino M, Marin D, Guerrisi A, Baski M, Galati F, Rossi M, Brozzetti S, Masciangelo R, Passariello R, Catalano C. Intraindividual comparison of gadoxetate disodium-enhanced MR imaging and 64-section multidetector CT in the Detection of hepatocellular carcinoma in patients with cirrhosis. Radiology 2010; 256: 806-16.

6. Heijmen L, Ter Voert EE, Nagtegaal ID, Span P, Bussink J, Punt CJ, de Wilt JH, Sweep FC, Heerschap A, van Laarhoven HW. Diffusion-weighted MR imaging in liver metastases of colorectal cancer: reproducibility and biological validation. Eur Radiol 2013; 23: 748-56.

7. Kele PG, van der Jagt EJ. Diffusion weighted imaging in the liver. World J Gastroenterol. 2010; 16: 1567-76.

8. Le Bihan D, Breton E, Lallemand D, Grenier P, Cabanis E, Laval-Jeantet M. MR imaging of intravoxel incoherent motions: application to diffusion and perfusion in neurologic disorders. Radiology 1986; 161:401-407.

9. Le Bihan D, Breton E, Lallemand D, Aubin ML, Vignaud J, Laval-Jeantet M. Separation of diffusion and perfusion in intravoxel incoherent motion MR imaging. Radiology 1988; 168:497-505.

10. Granata V, Fusco R, Catalano O, Filice S, Amato DM, Nasti G, Avallone A, Izzo F, Petrillo A. Early Assessment of Colorectal Cancer Patients with Liver Metastases Treated with Antiangiogenic Drugs: The Role of Intravoxel Incoherent Motion in Diffusion-Weighted Imaging. PLoS One 2015; 10: e0142876.

11. Granata V, Fusco R, Catalano O, Piccirillo M, De Bellis M, Izzo F, Petrillo A. Percutaneous ablation therapy of hepatocellular carcinoma with irreversible electroporation: MRI findings. AJR Am J Roentgenol 2015; 204: 1000-7.

12. Gupta N, Sureka B, Kumar MM, Malik A, Bhushan TB, Mohanty NK. Comparison of dynamic contrast-enhanced and diffusion weighted magnetic resonance image in staging and grading of carcinoma bladder with histopathological correlation. Urol Ann 2015; 7: 199-204.

13. Woo S, Cho JY, Kim SY, Kim SH. Histogram analysis of apparent diffusion coefficient map of diffusion-weighted MRI in endometrial cancer: a preliminary correlation study with histological grade. Acta Radiol 2014; 55: 1270-7.

14. Cipolla V, Santucci D, Guerrieri D, Drudi FM, Meggiorini ML, de Felice C. Correlation between 3T apparent diffusion coefficient values and grading of invasive breast carcinoma. Eur J Radiol 2014; 83: 2144-50.

15. Cuccarini V, Erbetta A, Farinotti M, Cuppini L, Ghielmetti F, Pollo B, Di Meco F, Grisoli M, Filippini G, Finocchiaro G, Bruzzone MG, Eoli M. Advanced MRI may complement histological diagnosis of lower grade gliomas and help in predicting survival. J Neurooncol 2016; 126: 279-88.

16. Guo W, Zhao S, Yang Y, Shao G. Histological grade of hepatocellular carcinoma predicted by quantitative diffusion-weighted imaging. Int J Clin Exp Med 2015; 8: 4164-9.

17. Woo S, Lee JM, Yoon JH, Joo I, Han JK, Choi BI. Intravoxel incoherent motion diffusion-weighted MR imaging of hepatocellular carcinoma: correlation with enhancement degree and histologic grade. Radiology 2014; 270: 758-67.

18. Chen J, Wu M, Liu R, Li S, Gao R, Song B. Preoperative evaluation of the histological grade of hepatocellular carcinoma with diffusion-weighted imaging: a metaanalysis. PLoS One 2015; 10: e0117661.

19. Li X, Li C, Wang R, Ren J, Yang J, Zhang Y. Combined Application of Gadoxetic Acid Disodium-Enhanced Magnetic Resonance Imaging (MRI) and DiffusionWeighted Imaging (DWI) in the Diagnosis of Chronic Liver Disease-Induced Hepatocellular Carcinoma: A MetaAnalysis. PLoS One 2015; 10: e0144247.

20. Zhou L, Rui JA, Wang SB, Chen SG, Qu, Q, Chi TY, Wei X, Han K, Zhang N, Zhao HT. Factors predictive for long-term survival of male patients with hepatocellular carcinoma after curative resection. J Surg Oncol; 95:298-303. 
21. Granata V, Catalano O, Fusco R, Tatangelo F, Rega D, Nasti G, Avallone A, Piccirillo M, Izzo F, Petrillo A. The target sign in colorectal liver metastases: an atypical Gd-EOBDTPA "uptake" on the hepatobiliary phase of MR imaging. Abdom Imaging 2015; 40: 2364-71.

22. Fusco R, Sansone M, Petrillo A. The Use of the LevenbergMarquardt and Variable Projection Curve-Fitting Algorithm in Intravoxel Incoherent Motion Method for DW-MRI Data Analysis. Applied Magnetic Resonance 2015; 46: 551-558.

23. Kim SH, Lim HK, Choi D, Lee WJ, Kim SH, Kim MJ, Kim CK, Jeon YH, Lee JM, Rhim H. Percutaneous radiofrequency ablation of hepatocellular carcinoma: effect of histologic grade on therapeutic results. Am J Roentgenol 2006; 186:S327-33.

24. Ludwig JM, Camacho JC, Kokabi N, Xing M, Kim HS. The Role of Diffusion-Weighted Imaging (DWI) in Locoregional Therapy Outcome Prediction and Response Assessment for Hepatocellular Carcinoma (HCC): The New Era of Functional Imaging Biomarkers. Pereira PL, ed. Diagnostics. 2015;5:546-563.

25. Nakanishi M, Chuma M, Hige S, Omatsu T, Yokoo H, Nakanishi K, Kamiyama T, Kubota K, Haga H, Matsuno Y, Onodera Y, Kato M, Asaka M. Relationship between diffusion-weighted magnetic resonance imaging and histological tumor grading ofhepatocellular carcinoma. Ann Surg Oncol. 201;19:1302-9.
26. Nasu K, Kuroki Y, Tsukamoto T, Nakajima H, Mori K, Minami M. Diffusion-weighted imaging of surgically resected hepatocellular carcinoma:imaging characteristics and relationship among signal intensity, apparent diffusion coefficient, and histopathologic grade. AJR Am J Roentgenol 2009; 193: 438-44.

27. Muhi A, Ichikawa T, Motosugi U, Sano K, Matsuda M, Kitamura T, Nakazawa T, Araki T. High-b-value diffusionweighted MR imaging of hepatocellular lesions: estimation of grade of malignancy of hepatocellular carcinoma. J Magn Reson Imaging 2009; 30: 1005-11.

28. Nishie A, Tajima T, Asayama Y, Ishigami K, Kakihara D, Nakayama T, Takayama Y, Okamoto D, Fujita N, Taketomi A, Yoshimitsu K, Honda H. Diagnostic performance of apparent diffusion coefficient for predicting histological grade of hepatocellular carcinoma. Eur J Radiol 2011; 80: e29-33.

29. Zhang JL, Sigmund EE, Rusinek H, Chandarana H, Storey P, Chen Q, Lee VS. Optimization of b-value sampling for diffusion-weighted imaging of the kidney. Magn Reson Med 2012; 67: 89-97

30. Koh DM, Collins DJ, Orton MR. Intravoxel incoherent motion in body diffusion-weighted MRI: reality and challenges. AJR Am J Roentgenol 2011; 196: 1351-61. 